University of Nebraska - Lincoln

DigitalCommons@University of Nebraska - Lincoln

Norman R. Simon Papers

Research Papers in Physics and Astronomy

$12-15-1985$

Fourier Decomposition of RR Lyrae Pulsations: Theory Versus

Observations

Norman R. Simon

University of Nebraska - Lincoln, nsimon@unl.edu

Follow this and additional works at: https://digitalcommons.unl.edu/physicssimon

Simon, Norman R., "Fourier Decomposition of RR Lyrae Pulsations: Theory Versus Observations" (1985). Norman R. Simon Papers. 20.

https://digitalcommons.unl.edu/physicssimon/20

This Article is brought to you for free and open access by the Research Papers in Physics and Astronomy at DigitalCommons@University of Nebraska - Lincoln. It has been accepted for inclusion in Norman R. Simon Papers by an authorized administrator of DigitalCommons@University of Nebraska - Lincoln. 
The AstrophysiCal Journal, 299:723-727, 1985 December 15

(C) 1985. The American Astronomical Society. All rights reserved. Printed in U.S.A.

\title{
FOURIER DECOMPOSITION OF RR LYRAE PULSATIONS: THEORY VERSUS OBSERVATIONS
}

\author{
N. R. SIMON \\ Department of Physics and Astronomy, University of Nebraska-Lincoln \\ Received 1985 March 25; accepted 1985 June 18
}

\begin{abstract}
Fourier decomposition is employed to compare the light curves of RR Lyrae stars with those emerging from hydrodynamic models. Very good agreement is obtained between theory and observation for the RR $c$ stars, but in the case of the RRab stars there are significant discrepancies in the Fourier phase quantities $\phi_{21}$ and $\phi_{31}$. The deficiencies of the models are not remedied by replacing Carson opacities with Los Alamos opacities, nor by including dynamic zoning in the hydrogen ionization region. We also Fourier analyze the theoretical velocity curves to determine $R_{21}, \phi_{21}$, and $\phi_{31}$ as well as the first-order phase lag $(\Delta \phi)_{1}$. It is suggested that $(\Delta \phi)_{1}$ may be correlated with surface temperature.

Subject headings: stars: interiors — stars: pulsation — stars: RR Lyrae
\end{abstract}

\section{INTRODUCTION}

The classical pulsating stars continue to play an important role not only in our understanding of stellar evolution, but also in the study of the history of our Galaxy and in extragalactic astronomy and cosmology as well. Detailed comparison of the light and velocity variations of these objects with the results from hydrodynamic models offers the opportunity to gain new information concerning the physical structure and evolutionary history of these interesting stars. The observed light curves of the classical pulsators have usually been described qualitatively in terms of "bumps," "dips," "shoulders," etc., or semiquantitatively with parameters measuring, for example, the asymmetry of the variations or the phase of some distinct feature. In addition, there is a venerable but not extensive history of the application to this problem of the quantitative technique of Fourier decomposition (see Payne-Gaposchkin 1947, and references therein).

The Fourier method seems to have fallen into disuse some four decades ago but was recently revived and refined, independently of the earlier work, by Simon and Lee (1981), who applied it to a large sample of light curves of classical Population I Cepheids. Subsequently, Fourier decomposition was employed to describe the velocity curves of classical Cepheids (Simon and Teays 1983) and the light curves of RR Lyrae stars in the field (Simon and Teays 1982) and in the globular cluster $\omega$ Centauri (Petersen 1984). On the theoretical side, Hodson, Cox, and King (1982) were the first to use Fourier analysis to treat hydrodynamic models, while Simon and Davis (1983) made the first comparison of models and observations employing the Fourier technique.

In the latter study, the light and velocity curves from a series of hydrodynamic models were compared with the classical Cepheid light curves treated by Simon and Lee (1981) and the velocity curves treated by Simon and Teays (1983). Serious discrepancies appeared between theory and observation-a result which is perhaps not surprising in view of the many well-known deficiencies in our picture of the classical Cepheids (e.g., Cox 1980). These problems include the failure of standard models to predict correct period ratios for the "bump" and "beat" Cepheids, and the inability of the hydrodynamic calculations to produce stable double-mode pulsation. One may contrast this case with that of the RR Lyrae stars. Although here, again, the nonlinear codes have not been able to show the double-mode phenomenon, the models do predict correct period ratios (Cox, Hodson, and Clancy 1983) with masses and physical assumptions consistent with accepted ideas regarding stellar evolution. Thus the RR Lyrae models are believed to be much more secure.

The purpose of the present investigation is to use Fourier decomposition to compare a set of nonlinear RR Lyrae calculations with the large observational sample studied by Simon and Teays (1982). This comparison is the analog of the SimonDavis investigation of the classical Cepheids. We shall find that while theory and observation seem to agree quite well for the $\mathrm{RR} c$ stars, there is a clear quantitative discrepancy concerning certain of the Fourier components in the RR $a b$ case. This discrepancy is potentially very useful insofar as it could point the way toward improved models.

\section{FOURIER DECOMPOSITION OF THE LIGHT CURVES}

All but two of the hydrodynamic models treated in this investigation were provided by $\mathrm{O}$. Hubickyj and R. Stothers. The sample consists of eight fundamental mode models constructed for the RRab stars (Stothers 1981) and seven first overtone models for the RRc stars (Hubickyj 1983). With one exception the Stothers-Hubickyj calculations were performed using the radiative opacities due to Carson (1976). Additional physical assumptions are discussed by Stothers (1981).

The methodology of the Stothers and Hubickyj studies was to integrate a grid of models from which suitable members could be selected to characterize real stars. Stothers (1981) published detailed comparisons between his models and the observations on such points as total amplitude, asymmetry, phase of a secondary bump, etc. Good agreement was found for certain models, leading to the identification of their parameters with those of the R Rab pulsators. However, in view of the past utility of the Fourier technique in providing an objective and independent characterization of pulsational variations, it was decided to apply this method as well.

Fourier decompositions were made of the StothersHubickyj light and velocity curves in a manner that allows direct comparison with observations (Simon and Davis 1983). The Fourier series had the form $A_{0}+A_{k} \cos \left(k \omega t+\phi_{k}\right)$ (summation convention) for the light, and $A_{0}-A_{k} \sin (k \omega t$ 


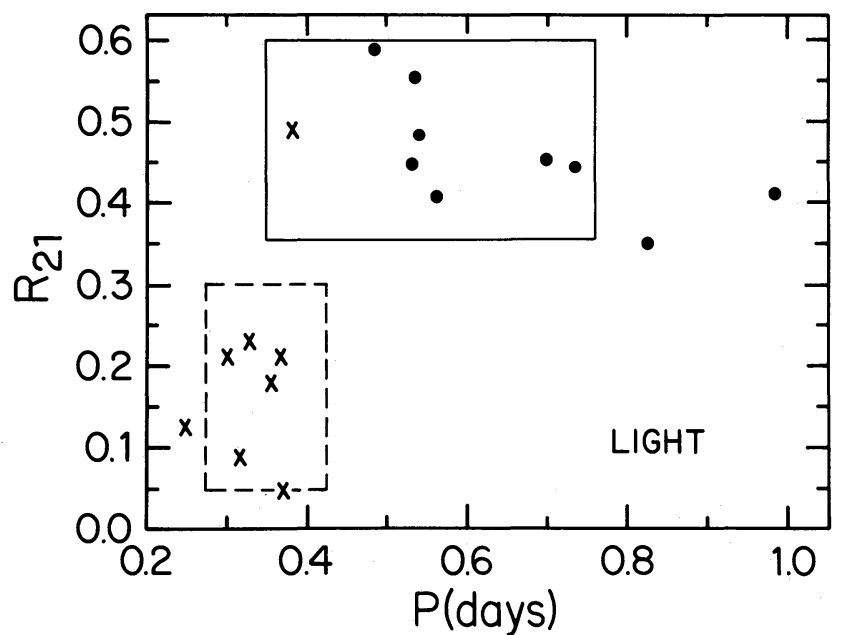

Fig. 1.-Fourier amplitude ratio $R_{21}$ vs. period for the light curves. Theoretical models-circles, fundamental mode; crosses, first overtone. Domains of observed stars—solid box, RR $a b$; dashed box, R R $c$.

$+\phi_{k}$ ) for the velocity. The amplitude ratios and phase differences are defined as follows: $R_{k 1}=A_{k} / A_{1}, \phi_{k 1}=\phi_{k}-k \phi_{1}$.

Since bolometric corrections for the RR Lyrae stars are quite small, the theoretical quantity $M_{\mathrm{BOL}}$ may be compared with observed $V$ magnitudes with little error. Figure 1 shows a plot of the amplitude ratio $R_{21}$ (Simon and Davis 1983) versus period for the light curves. Boxes indicate the domains of the observed stars (Simon and Teays 1982)-solid for the RRab type, dotted for the RRc. The fundamental mode models are denoted by dots, the overtone models by crosses. The agreement between theory and observation in Figure 1 is very good indeed. One notices only a single divergent point, an overtone model falling among the RR $a b$ stars.

In Figures 2 and 3 we plot against period the phase quantities $\phi_{21}$ and $\phi_{31}$ respectively. Circles surrounding some of the crosses indicate that these points were determined only marginally. In both figures there is again very good agreement between the overtone models (crosses) and the observed $\mathrm{RR} c$ stars (dashed boxes). However, the situation is quite different when one considers the fundamental mode. Here there emerges a large and distinct difference between theory and observa-

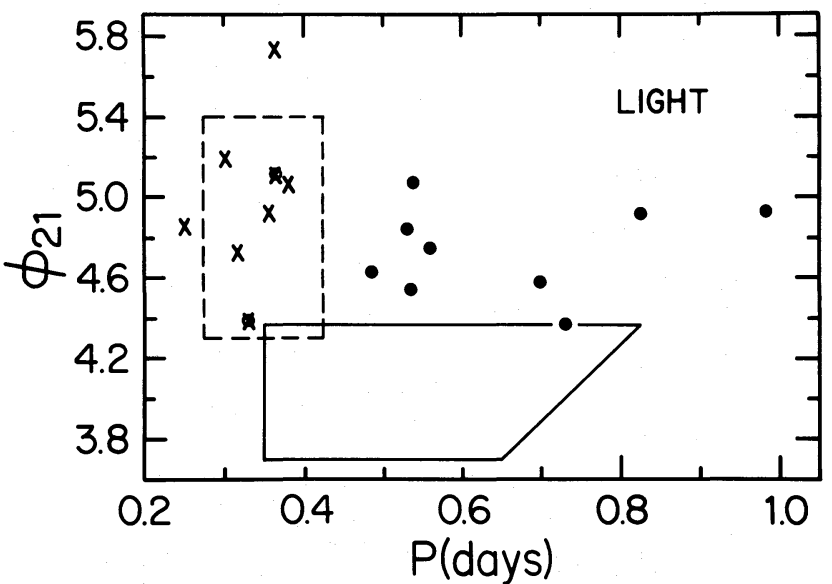

FIG. 2.-Fourier phase difference $\phi_{21}$ vs. period for the light curves. Notation as in Fig. 1. Circle surrounding a cross indicates that point is marginally determined.

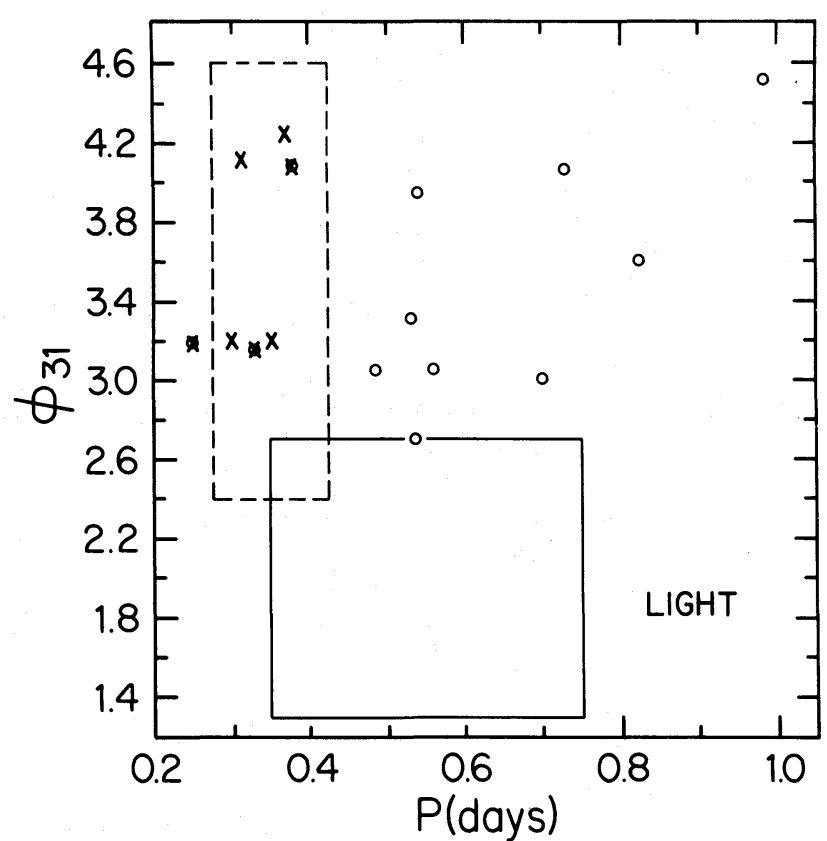

FIG. 3.-Fourier phase difference $\phi_{31}$ vs. period for the light curves. Notation as in Fig. 2

tions, with the fundamental mode models (circles) lying far above the observed RRab stars (solid boxes) in both diagrams. Taking crude eyeball averages, we find $\left\langle\phi_{21}\right\rangle_{\text {theor }} \approx 4.8$, $\left\langle\phi_{21}\right\rangle_{\text {obs }} \approx 4.1$, and $\left\langle\phi_{31}\right\rangle_{\text {theor }} \approx 3.4,\left\langle\phi_{31}\right\rangle_{\text {obs }} \approx 2.0$.

Although the Fourier coefficients provide a quantitative description of the light curve, it is also interesting to inquire as to the effects of differences in $\phi_{21}$ and $\phi_{31}$ on the light curve shapes. One gross property of the light curve that seems to be affected by these quantities is the width at half-maximum. We have examined the Stothers and Hubickyj light curves as well as a representative sample of $\mathrm{RR} a b$ and $\mathrm{RR} c$ stars from the observations of Lub (1977) and determined, at half-maximum, the fractional width $w$ of the light curve, defined as: extent at half-maximum light/total period. We find for the R R $a b$ stars: $0.33<w_{\text {theor }}<0.78, \quad \bar{w}_{\text {theor }}=0.54, \quad$ and $0.29<w_{\text {obs }}<0.32$, $\bar{w}_{\text {obs }}=0.30$; and for the $\operatorname{RR} c$ stars: $0.48<w_{\text {theor }}<0.68$, $\bar{w}_{\text {theor }}=0.53$, and $0.45<w_{\text {obs }}<0.68, \bar{w}_{\text {obs }}=0.55$. Thus the higher values of $\phi_{21}$ and $\phi_{31}$ seem to coincide with light curves that are broader at half-maximum. We may note here again the agreement between theory and observation for the $\mathrm{RR} c$ stars and the discrepancy in the R R $a b$ case.

The correspondence between $w$ and the Fourier phases is not precise, because the amplitudes of the Fourier terms also determine the shape of the light curve. The separate effect of the phases can be studied by constructing synthetic curves. We have put together two such light curves according to the form: $0.410 \cos \omega t+0.205 \cos \left(2 \omega t+\phi_{2}\right)+0.127 \cos \left(3 \omega t+\phi_{3}\right)$. The amplitudes chosen are typical of observed RRab stars, while the two pairs of phases we used correspond approximately to averages for the observed and theoretical light curves, respectively, i.e., (a) $\phi_{21}=4.0, \phi_{31}=2.0$ and $(b) \phi_{21}=$ $4.8, \phi_{31}=3.3$. Figure 4 shows the synthetic light curves for these two cases. The greater breadth of curve $b$ is readily apparent, while a measurement yields $w($ case $a) \approx 0.33, w($ case $b) \approx 0.53$. These values are in good agreement with those presented above for the observed and theoretical RRab light curves respectively. 

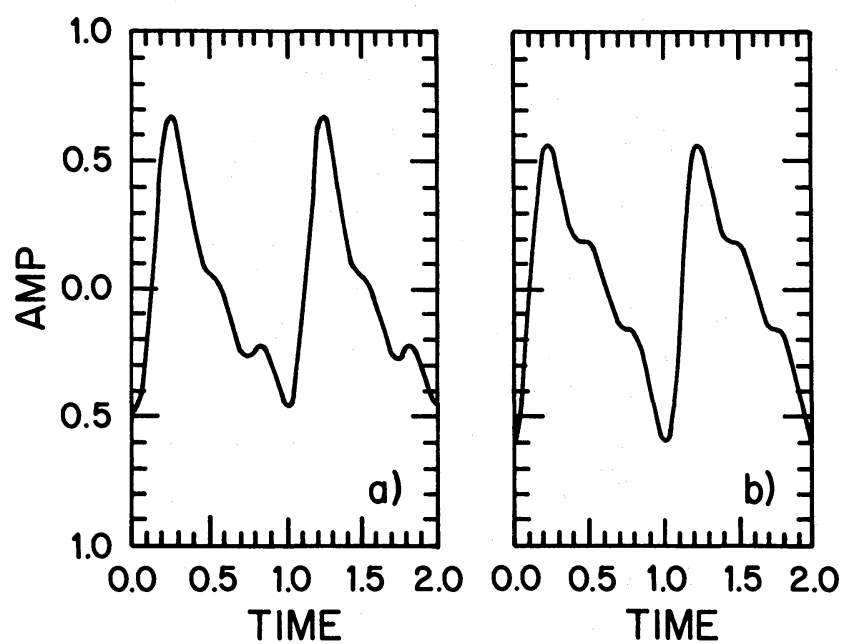

FIG. 4.- Synthetic light curves for RR $a b$ stars (see text). (a) $\phi_{21}=4.0$, $\phi_{31}=2.0 ;(b) \phi_{21}=4.8, \phi_{31}=3.3$.

Because the amplitudes were held fixed in this experiment, we can be sure that the difference in $w$ was due to the phases. However, it is far from clear that the width at half-maximum is the best parameter for contrasting the synthetic curves. For example, we might just as easily have made the comparison in terms of bump phases or of the width at some other point on the light curve. In our opinion, this ambiguity provides still another justification for preferring the Fourier description of the light curve.

\section{THE PHASE DISCREPANCY}

The difference noted between observed and calculated values of $\phi_{21}$ and $\phi_{31}$ for fundamental mode pulsators is significant. In the first place, the observed values are defined extremely well by the large sample of stars treated by Simon and Teays (1982). Second, not only is it true that the observed and theoretical domains do not overlap, but the difference in mean values between them is as large or larger than the extent of the domains themselves. And, finally, the theoretical values of the phase quantities considerably exceed the observed values over the whole range of RR $a b$ periods from about 0.35 days to about 0.8 days. This means that any changes made in the calculations to accommodate these differences would have to affect a wide range of models all in the same sense.

Why do the theoretical light curves display wrong values of $\phi_{21}$ and $\phi_{31}$ ? One possibility that must be considered is the use in the models of the Carson opacities. However, one of the fundamental mode points (circles) in Figures 1-3 represents a model calculated by Stothers (1981) employing Los Alamos opacities. This point lies among the others. To explore this matter further, a new hydrodynamic model has been constructed at Nebraska using Los Alamos opacities according to the fit of Stellingwerf (1975). This model has the following parameters: $\quad M=0.58 M_{\odot}, \quad L=39.2 L_{\odot}, \quad T_{e}=6400 \mathrm{~K}$, $X=0.70, Z=0.001, P_{\text {fund }}=0.56$ days. Fourier components of the light curve have also been included in Figures $1-3$ and, once more, this point lies among the others. We conclude that replacement of the Carson opacities with the Los Alamos version will not correct the phase discrepancies.

For completeness we have also calculated a first-overtone model with the same mass, luminosity, composition, and physical assumptions as above but at a somewhat higher tem-

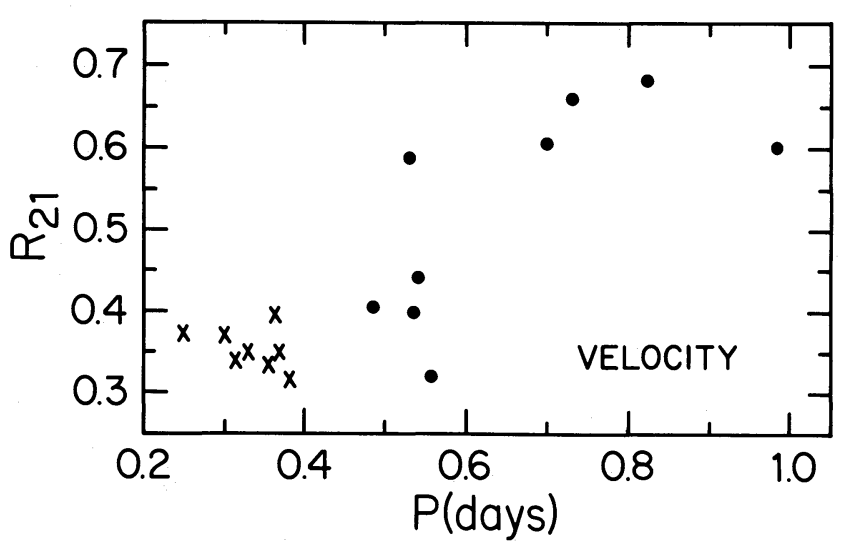

FIG. 5. $-R_{21}$ vs. period for the theoretical velocity curves. Circles, fundamental mode; crosses: first overtone.

perature, $T=6700 \mathrm{~K}$. The period of this model was $P_{\text {over }}=$ 0.36 days, and its Fourier coefficients also fell among those of the Carson-opacity models in Figures 1-3. It is interesting to note that the Nebraska models were integrated with a "temperature grid" code (Aikawa and Simon 1983), which included dynamic zoning of the hydrogen ionization region (HIR) similar to that of the DYN code (Castor, Davis, and Davison 1977). Thus the high values of $\phi_{21}$ and $\phi_{31}$ obtained in the fundamental mode models of Stothers (1981) do not seem attributable to relatively crude treatment of the HIR.

\section{VELOCITY CURVES AND PHASE LAGS}

Figures 5, 6, and 7 show plots versus period of the quantities $R_{21}, \phi_{21}$, and $\phi_{31}$ respectively for the hydrodynamic velocity curves (for notation, see Simon and Davis 1983). Unfortunately, the observational data presently in the literature are not accurate nor extensive enough for Fourier decomposition. Thus the theoretical velocity plots can be presented merely as predictions to be compared with suitable observations as they become available. It has recently come to our attention (W. Benz, private communication) that such observations are now underway.

In their study of classical Cepheids, Simon and Davis (1983) introduced the first-order phase lag, $(\Delta \phi)_{1}$. This quantity represents the difference (in radians) between maximum light and maximum velocity of expansion as defined not by the full light and velocity curves but rather by the relative phases of the first-order terms in the respective Fourier decompositions. Subsequently, Simon (1984) determined $(\Delta \phi)_{1}$ for each of a small group of short-period Population I Cepheids. It was found for these stars that maximum expansion velocity lagged

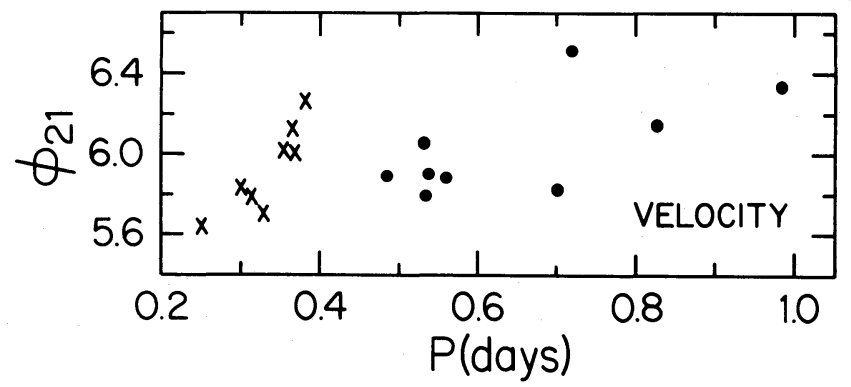

FIG. 6. $-\phi_{21}$ vs. period for the theoretical velocity curves. Notation as in Fig. 5. 


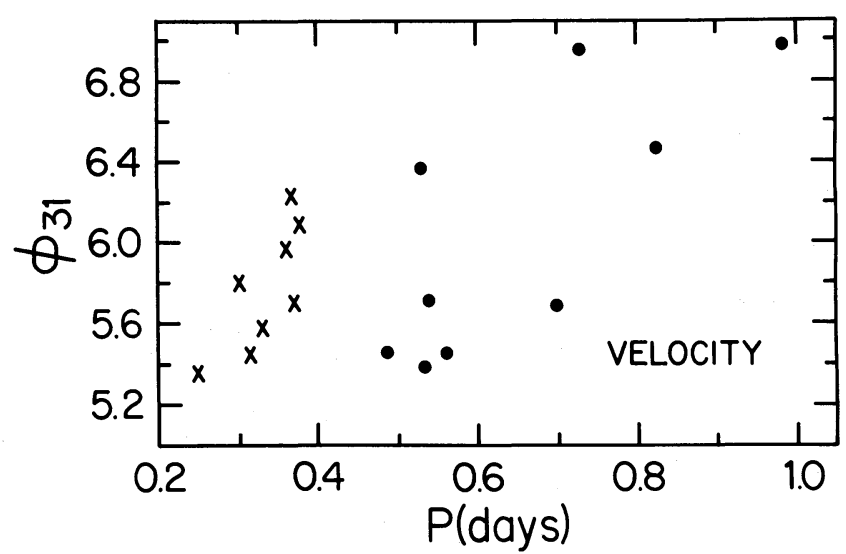

FIG. 7. $-\phi_{31}$ vs. period for the theoretical velocity curves. Notation as in Fig. 5.

maximum light on the average by about $0.3 \mathrm{rad}\left[\right.$ i.e., $(\Delta \phi)_{1} \approx$ $-0.3]$ in both the observations and the models, and that the two suspected overtone pulsators among the observed stars showed the most negative values of $(\Delta \phi)_{1}$.

In Figure 8 we plot the first-order phase lag $(\Delta \phi)_{1}$ against period for the theoretical calculations of the present study. Although there is some overlap, one may note the tendency for the overtone models to show smaller values of $(\Delta \phi)_{1}$ than do the fundamental mode models. However, the latter models all have positive phase lags indicating that, in first order, the velocity leads rather than lags the light. This is contrary to the situation in the classical Cepheids. We may also compare this result with the full nonlinear phase lags for the same models. These were obtained by Stothers (1981), who found that the velocity curve led the light in some cases but lagged in others. A precise observational determination of this question must await new data.

Returning to Figure 8 , we note a considerable amount of scatter in the first-order phase lag. Certainly there is a lack of any smooth progression with period. This circumstance leads us to inquire whether an additional parameter might govern $(\Delta \phi)_{1}$. Figure 9 displays a graph of the first-order phase lag versus temperature for the theoretical models. One sees here a clear tendency for $(\Delta \phi)_{1}$ to increase with decreasing temperature, irrespective of the oscillation mode. We have reexamined the classical Cepheid models of Simon and Davis (1983)

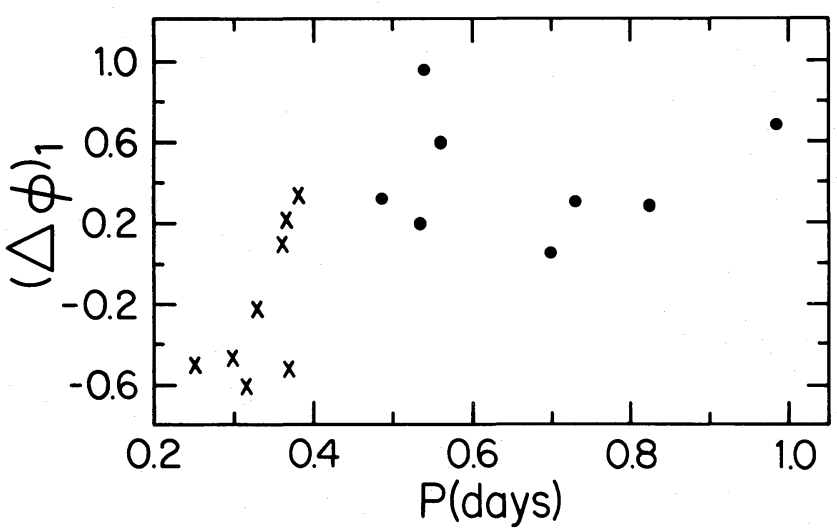

Fig. 8.-The first-order phase lag $(\Delta \phi)_{1}$ vs. period for the theoretical models. Notation as in Fig. 5.

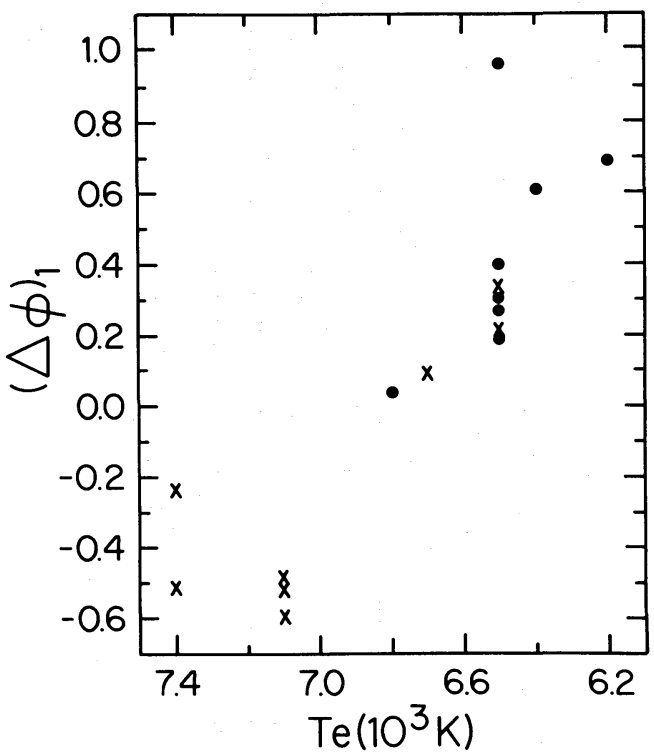

FIG. 9. $-(\Delta \phi)_{1}$ vs. effective temperature (units of $10^{3} \mathrm{~K}$ ) for the theoretical models. Notation as in Fig. 5.

to look for a similar trend but have not found it. Neither does it appear in the full nonlinear phase lags of Stothers (1981). Nonetheless, this effect should receive more thorough examination in further models. Clearly, if it could be established that a correlation exists between $(\Delta \phi)_{1}$ and temperature, this fact could be of considerable value in the study of pulsating stars.

\section{DISCUSSION}

In this investigation we have employed the technique of Fourier decomposition to compare observed and calculated light curves of RR Lyrae pulsators. Very good agreement has been found for the RRc stars. However, for the RR $a b$ stars, a quantitative discrepancy between models and observations is reflected in the Fourier phase quantities $\phi_{21}$ and $\phi_{31}$. The calculated values of these quantities are considerably larger than the values determined from observed stars.

We have found that this disagreement is not changed when Los Alamos opacities are substituted for Carson opacities, nor is it ameliorated by the inclusion of dynamic zoning in the hydrogen ionization region. At the moment it is not clear how drastic a change would be necessary in the models in order to substantially narrow the differences in $\phi_{21}$ and $\phi_{31}$. Because these quantities are well defined and can be determined in a straightforward manner, the possibility arises of effecting meaningful improvement in the hydrodynamic codes by seeking alterations which place the theoretical light curves into conformity with the observations.

Thus, a number of questions come to mind: Is there a preferred mass-luminosity relation with respect to the values of $\phi_{21}$ and $\phi_{31}$ ? Could changes in the opacity law or chemical composition move the Fourier phase quantities in the proper direction? What is the role in this regard of the amount and form of artificial viscosity in the models? How would the inclusion of convection affect the details of the light curves? Might the presence of atmospheric shocks influence the Fourier phases?

It is clear that progress on these questions and others will require a new series of hydrodynamic calculations. At the same 
time, radial velocity observations of RR Lyrae stars are needed to provide data that can be compared with theoretical velocity curves and phase lags. There is a great deal still to be learned regarding RR Lyrae pulsators, but the opportunity to do so seems now at hand.
The author is grateful to O. Hubickyj and R. Stothers for providing light and velocity curves from their hydrodynamic calculations. Without their efforts this study could not have been undertaken. It is also a pleasure to thank T. Aikawa for integrating two nonlinear, Los Alamos opacity models. This work was partially funded by the National Science Foundation under grant AST 83-16875.
Aikawa, T., and Simon, N. R. 1983, Ap. J., 273, 346.

Carson, T. R. 1976, Ann. Rev. Astr. Ap., 14, 95.

Castor, J. I., Davis, C. G., and Davison, D. K. 1977, Los Alamos Scientific Lab. Rept., No. LA-6664.

Cox, A. N. 1980, Ann. Rev. Astr. Ap., 18, 15.

Cox, A. N., Hodson, S. W., and Clancy, S. P. 1983, Ap. J., 266, 94.

Hodson, S. W., Cox, A. N., and King, D. S. 1982, Ap. J., 253, 260.

Hubickyj, O. 1983, Ph.D. thesis, City University of New York.

Lub, J. 1977, Astr. Ap. Suppl., 29, 345.

\section{REFERENCES}

Payne-Gaposchkin, C. 1947, A.J., 52, 218.

Petersen, J. O. 1984, Astr. Ap., 139, 496

Simon, N. R. 1984, Ap. J., 284, 178.

Simon, N. R., and Davis, C. G. 1983, Ap. J. 266, 787.

Simon, N. R., and Lee, A. S. 1981, Ap. J., 248, 291

Simon, N. R., and Teays, T. J. 1982, Ap. J., 261, 586

. 1983, Ap. J., 265, 997.

Stellingwerf, R. F. 1975, Ap. J., 195, 441.

Stothers, R. 1981, Ap. J., 247, 941

NORMAN R. SIMON: Behlen Laboratory of Physics, University of Nebraska, Lincoln, NE $\quad 68588-0111$ 